\title{
Neuroinflammation in Schizophrenia-Related Psychosis: A PET Study
}

\author{
Janine Doorduin ${ }^{1}$, Erik F.J. de Vries ${ }^{1}$, Antoon T.M. Willemsen ${ }^{1}$, Jan Cees de Groot ${ }^{2}$, Rudi A. Dierckx ${ }^{1}$,
} and Hans C. Klein ${ }^{1,3}$

${ }^{I}$ Department of Nuclear Medicine and Molecular Imaging, University Medical Center Groningen, University of Groningen, Groningen, The Netherlands; ${ }^{2}$ Department of Radiology, University Medical Center Groningen, University of Groningen, Groningen, The Netherlands; and ${ }^{3}$ University Center of Psychiatry, University Medical Center Groningen, University of Groningen, Groningen, The Netherlands

Schizophrenia is a chronic and disabling brain disease characterized by psychotic episodes with unknown etiology. It is suggested that neuroinflammation plays a role in the pathophysiology of schizophrenia. Neuroinflammation is characterized by the activation of microglia cells, which show an increase in the expression of the peripheral benzodiazepine receptor. The isoquinoline $(R)-N-{ }^{11} \mathrm{C}$-methyl- $N$-(1-methylpropyl)-1-(2-chlorophenyl)isoquinoline-3-carboxamide $\left({ }^{11} \mathrm{C}-(R)-\mathrm{PK} 11195\right)$ is a peripheral benzodiazepine receptor ligand that can be used for the imaging of activated microglia cells, and thus neuroinflammation, with PET. We hypothesized that neuroinflammation would be more profound in schizophrenic patients during psychosis, and it was therefore investigated whether neuroinflammation was present in patients within the schizophrenia spectrum who were in a psychotic phase. Methods: Seven patients within the schizophrenia spectrum who were recovering from psychosis were included. Recovering psychosis was defined by a score of 5 or more on 1 item of the positive scale of the positive and negative symptoms scale (PANSS) or a score of 4 on 2 items. The patients were compared with 8 age-matched healthy volunteers. Dynamic 60-min PET scans were acquired after the injection of ${ }^{11} \mathrm{C}-(R)-\mathrm{PK} 11195$. All subjects underwent T1- and T2-weighted MRI, and the scans were visually examined for abnormalities and used for anatomic coregistration in data analysis. The PET data were analyzed with a 2-tissuecompartment model to calculate the binding potential, using the metabolite-corrected plasma curve as input. Results: A significantly higher binding potential of ${ }^{11} \mathrm{C}-(R)-\mathrm{PK} 11195$, indicative of neuroinflammation, was found in the hippocampus of schizophrenic patients than in healthy volunteers $(2.07 \pm 0.42$ vs. 1.37 $\pm 0.30 ; P=0.004)$. A nonsignificant $30 \%$ higher ${ }^{11} \mathrm{C}-(R)-$ PK11195 binding potential was found in the whole-brain gray matter of schizophrenic patients. The MR images did not reveal any visual abnormalities. Conclusion: The present study suggests that focal neuroinflammation may play an important role in schizophrenia during psychosis.

Received May 28, 2009; revision accepted Aug. 11, 2009.

For correspondence or reprints contact: Janine Doorduin, Department of Nuclear Medicine and Molecular Imaging, University Medical Center Groningen, University of Groningen, P.O. Box 30.001, 9700 RB Groningen, The Netherlands.

E-mail: j.doorduin@ngmb.umcg.nl

COPYRIGHT () 2009 by the Society of Nuclear Medicine, Inc.
Key Words: schizophrenia; psychosis; neuroinflammation; microglia; PET; ${ }^{11} \mathrm{C}-\mathrm{PK} 11195$

J Nucl Med 2009; 50:1801-1807

DOI: 10.2967/jnumed.109.066647

\section{O}

chizophrenia is a chronic, disabling brain disease accompanied by psychosis with positive symptoms, such as hallucinations and delusions. Despite considerable research, the exact etiology of psychosis remains unknown. Disturbances in immune mechanisms are thought to play an important role in the psychosis of schizophrenia (1). Although these disturbances in immune mechanisms were mainly found in peripheral blood and in cerebrospinal fluid, they were hypothesized to derive from inflammatory processes in the central nervous system. Indeed, evidence from postmortem studies suggests that schizophrenia is associated with an increased number of activated microglia cells.

Microglia cells are the predominant population of macrophages in the brain and are responsive to injury or infection of brain tissue. In healthy brain tissue, microglia cells have a ramified morphology, characterized by long processes that continuously survey the microenvironment (2). In response to brain injury or infection, microglia cells change from the ramified morphology into a reactive or amoeboid form. Activated microglia cells, characteristic of neuroinflammation, are involved in the removal of the infectious agents and irreversibly damaged brain tissue. However, in neurologic disorders this process runs out of control, resulting in chronic microglia cell activation, which has a detrimental effect. Although neuroinflammation has been shown to play a major role in many neurodegenerative diseases, such as multiple sclerosis, Parkinson disease, and Alzheimer disease (3), only limited and ambiguous data on the presence of neuroinflammation in psychiatric diseases such as schizophrenia exist.

Postmortem studies in schizophrenic patients have demonstrated the presence of activated microglia cells in the 
brain. However, the results of these studies are inconsistent. Some studies showed an increased density of microglia cells in a subpopulation of schizophrenic patients $(4,5)$, but other studies could not provide evidence for such an increase $(6,7)$. This might be explained by the differences in markers used for microglia cells and the differences in brain regions that were examined.

Thus far, most of the findings supporting the presence of neuroinflammation in schizophrenia are derived from postmortem studies in a limited number of brain areas, without the specific selection of patients with psychosis. PET provides the opportunity to study the presence of neuroinflammation in psychotic patients noninvasively. In neuroinflammation, activated microglia cells exhibit an increase in the expression of peripheral benzodiazepine receptors (PBRs) in the outer mitochondrial membrane. The PET tracer $(R)-N-{ }^{11} \mathrm{C}$-methyl- $N$-(1-methylpropyl)1-(2-chlorophenyl)isoquinoline-3-carboxamide $\quad\left({ }^{11} \mathrm{C}-(R)\right.$ PK11195) is an antagonist of the PBR, and binding of ${ }^{11} \mathrm{C}-(R)-\mathrm{PK} 11195$ to the PBR can be used to visualize neuroinflammation. ${ }^{11} \mathrm{C}-(R)-\mathrm{PK} 11195$ has already been used to show the presence of neuroinflammation in neurologic diseases, such as Parkinson disease, Alzheimer disease, multiple sclerosis, and herpes encephalitis (3). A recent study also showed a general increase in the whole-brain gray matter binding of ${ }^{11} \mathrm{C}-(R)-\mathrm{PK} 11195$ in schizophrenic patients within the first $5 \mathrm{y}$ of disease onset (8). No specific foci of neuroinflammation were observed. We hypothesized that microglia cells would be more active in schizophrenic patients during psychosis. In the present study, we investigated whether ${ }^{11} \mathrm{C}-(R)-\mathrm{PK} 11195$ could demonstrate the presence of neuroinflammation in patients within the schizophrenia spectrum who were in a psychotic phase.

\section{MATERIALS AND METHODS}

\section{Subjects}

On the basis of the following inclusion criteria, 10 patients were recruited from local psychiatric hospitals: fulfillment of DSM-IV criteria for the schizophrenia spectrum (295.xx and 298.xx) (9); psychosis, that is, a total score of 14 or higher on the positive scale of the positive and negative symptoms scale (PANSS) and at least a score of 5 on 1 item or a score of 4 on 2 items of the positive scale of the PANSS; age above $18 \mathrm{y}$; no use of benzodiazepines within 3 half-lives (on average, 1-2 wk) of the benzodiazepines before the start of the study; and the ability to provide written informed consent. Healthy volunteers, matched for age and sex, were recruited by advertisement and were included if they had no personal history of psychiatric disorders, no family history of psychiatric disorders in their first-degree relatives, and no presence of inflammation as measured by C-reactive protein (CRP) levels (i.e., CRP $<0.5 \mathrm{mg} / \mathrm{L}$ ). The exclusion criteria for all subjects were concomitant or past severe medical conditions, substance abuse, the use of nonsteroidal antiinflammatory drugs or paracetamol, pregnancy, and the presence of irremovable magnetic materials in or on the body. Diagnoses were classified by an experienced psychiatrist using the Schedule for Clinical Assessment in Neuropsychiatry, version 2 (SCAN2; World Health
Organization). Psychopathology in patients was assessed with the PANSS by trained psychiatric nurses.

Of the 20 included subjects, 3 patients and 2 healthy volunteers were excluded. One patient withdrew from the study after MRI was performed, so no ${ }^{11} \mathrm{C}-(R)$-PK11195 PET scan was acquired. A second patient had too much head movement during the MRI scan, and consequently the MRI scan could not be used for normalization of the PET data. A third patient had enlarged ventricles within the physiologic range, which hampered normalization of the PET scan. For the 2 healthy volunteers, the PET scans were not obtained because the arterial catheter could not be placed. No healthy volunteers were excluded because of elevated CRP.

The study was approved by the medical ethical committee of the University Medical Center Groningen. All subjects provided written informed consent after receiving a complete description of the study.

\section{Radiochemistry}

${ }^{11} \mathrm{C}-(R)$-PK11195 was labeled by trapping ${ }^{11} \mathrm{C}$-methyl iodide in a solution of $1 \mathrm{mg}$ of $(R)-N$-desmethyl-PK11195 and $10 \mathrm{mg}$ of potassium hydroxide in $300 \mu \mathrm{L}$ of dimethylsulfoxide. The reaction mixture was allowed to react for $1 \mathrm{~min}$ at $40^{\circ} \mathrm{C}$, neutralized with $1 \mathrm{M} \mathrm{HCl}$, and passed through a 45- $\mu \mathrm{m}$ Millex-HV filter (Millipore). The filtrate was purified by high-performance liquid chromatography (HPLC) using a $\mu$ Bondapak C18 column $(7.8 \times$ $300 \mathrm{~mm}$ ) (Waters) with acetonitrile/25 mM NaH${ }_{2} \mathrm{PO}_{4}$ (pH 3.5; 55/ 45 ) as the eluent (flow, $5 \mathrm{~mL} / \mathrm{min}$ ). To remove the organic solvents from the product, the collected HPLC fraction (retention time, 7 min) was diluted with $100 \mathrm{~mL}$ of water and passed through an Oasis HLB 30-mg (1 mL) cartridge. The cartridge was washed twice with $10 \mathrm{~mL}$ of water and subsequently eluted with $1 \mathrm{~mL}$ of ethanol and $8 \mathrm{~mL}$ of water. The product was sterilized by filtration over a $0.20-\mu \mathrm{m}$ Millex-LG filter (Millipore). The product was obtained in a $36 \% \pm 12 \%$ radiochemical yield $(n=16)$. Quality control was performed by HPLC, using a Novapak C18 column $(150 \times 3.9 \mathrm{~mm})$ (Waters) with acetonitrile/25 mM NaH $\mathrm{mO}_{4}(\mathrm{pH}$ $3.5)(60 / 40)$ as the eluent at a flow of $1 \mathrm{~mL} / \mathrm{min}$. The radiochemical purity was always greater than $95 \%$, and the specific activity was $89 \pm 58 \mathrm{GBq} / \mu \mathrm{mol}$. No differences were found between the injected dose in healthy volunteers $(398 \pm 38 \mathrm{MBq})$ and that in patients $(398 \pm 61)(P=0.995)$. The injected mass was slightly higher in patients than in healthy volunteers $(1.9 \pm 1.5$ vs. $0.7 \pm$ $0.4 \mathrm{mg} / \mathrm{L}, P=0.051)$ because of a lower specific activity in patients $(56 \pm 42 \mathrm{GBq} / \mu \mathrm{mol}$ vs. $112 \pm 58 \mathrm{GBq} / \mu \mathrm{mol}, P=0.053)$.

\section{PET Protocol}

A catheter was inserted in the radial artery after testing for collateral circulation with the Allen test and injection of $1 \%$ lidocaine (Fresenius Kabi Nederland BV) for local anesthesia. In the other arm, a catheter was inserted in the antebrachial vein. PET was performed with the ECAT EXACT HR + camera (Siemens). Head movement was minimized with a head-restraining adhesive band, and a neuroshield was used to minimize the interference of radiation from the subject's body. A 60-min emission scan in 3-dimensional (3D) mode was obtained, starting simultaneously with the intravenous injection of ${ }^{11} \mathrm{C}-(R)-\mathrm{PK} 11195$. The tracer was injected at a speed of $0.5 \mathrm{~mL} / \mathrm{s}$ (total volume, $8.3 \mathrm{~mL}$ ).

After radiotracer injection, arterial blood radioactivity was continuously monitored with an automated sampling system (Veenstra Instruments). Five extra blood samples were collected 
at $10,20,30,45$, and $60 \mathrm{~min}$ after ${ }^{11} \mathrm{C}-(R)-\mathrm{PK} 11195$ injection to determine the amount of radioactivity in the blood and plasma, to calibrate the sampling system. The arterial blood samples that were collected at 20, 45, and $60 \mathrm{~min}$ after ${ }^{11} \mathrm{C}-(R)$-PK11195 injection were also used for metabolite analysis. These blood samples were centrifuged at $3,000 \mathrm{~g}$ for $3 \mathrm{~min}$, and $1.5 \mathrm{~mL}$ of plasma were collected. Then $2 \mathrm{~mL}$ of acetonitrile (Rathburn Chemicals Ltd.) was added to the plasma to precipitate plasma proteins. The plasma samples were shaken on a vortex mixer for $30 \mathrm{~s}$ and centrifuged at $3,000 \mathrm{~g}$ for $5 \mathrm{~min}$. A volume of $1 \mathrm{~mL}$ of the supernatant was injected into an HPLC system, consisting of a 590 HPLC pump (Waters) and an Alltima 5- $\mu$ m RP-C ${ }_{18}$ column (inner diameter, $250 \times 10.0 \mathrm{~mm}$ ) (Alltech). The mobile phase consisted of a mixture of $69.5 \%$ acetonitrile (Rathburn Chemicals Ltd.), $30 \%$ water (Fresenius Kabi), and $0.5 \%$ triethylamine (Merck). The flow rate was set at $5.0 \mathrm{~mL} / \mathrm{min}$, and samples were collected at intervals of $30 \mathrm{~s}$. The collected samples were counted for radioactivity using a $\gamma$-counter (LKB Wallac).

\section{MRI}

All subjects underwent MRI of the brain using a 3-T Intera MRI scanner (Philips). The following images were obtained in alignment with the anterior commissure-posterior commissure plane: transaxial 3D T1-weighted gradient-echo images (repetition time [RT], $25 \mathrm{~ms}$; echo time [ET], $4.6 \mathrm{~ms}$; field of view [FOV], $256 \times 160 \times 204 \mathrm{~mm}$; matrix, $265 \times 265$; slice thickness, $2.0 \mathrm{~mm}$; 160 slices; 1 average; $\alpha=30^{\circ}$ ), 3D T1-weighted turbo gradientecho images (RT, $7.5 \mathrm{~ms}$; ET, $3.2 \mathrm{~ms}$; FOV, $260 \times 160 \times 232.14$ $\mathrm{mm}$; matrix, $265 \times 265$; slice thickness, $1.0 \mathrm{~mm}$; 160 slices; 1 average; $\alpha=8^{\circ}$ ), dual-echo images (RT, 3,000 ms; ET, 26.7 and $120 \mathrm{~ms}$; inversion time, $2,800 \mathrm{~ms}$; FOV, $220 \times 150 \times 175.31 \mathrm{~mm}$; matrix, $265 \times 265$; slice thickness, $3.0 \mathrm{~mm}$; 50 slices; 1 average; $\alpha=90^{\circ}$ ), and $\mathrm{T} 2$-weighted fluid-attenuated inversion recovery images (RT, 11,000 ms; ET, $100 \mathrm{~ms}$; inversion time, 2,800 ms; FOV, $220 \times 150 \times 175.31 \mathrm{~mm}$; matrix, $265 \times 265$; slice thickness, $3.0 \mathrm{~mm}$; 50 slices; 2 averages; $\alpha=90^{\circ}$ ). The 3D T1-weighted gradient-echo MR images were used to align to the PET image for normalization. The MR images of all subjects were visually examined for structural abnormalities and neuroinflammation by an experienced neuroradiologist.

\section{Data Analysis}

Attenuation correction was performed with the separate ellipse algorithm. Images were reconstructed by filtered backprojection in 21 successive frames of increasing duration $(6 \times 10 \mathrm{~s}, 2 \times 30 \mathrm{~s}, 3 \times$ $1 \mathrm{~min}, 2 \times 2 \mathrm{~min}, 2 \times 3 \mathrm{~min}, 3 \times 5 \mathrm{~min}$, and $3 \times 10 \mathrm{~min})$. The first 2 min of the PET scan were summed to create an image that resembled a perfusion image, which was used to align the MR image to the corresponding PET image using Statistical Parametric Mapping (SPM2) software (Wellcome Department of Imaging Neuroscience). The aligned MR image was normalized to the SPM2 MRI template. The normalization was then applied to each individual frame of the PET image. A limited number of brain regions of interest (ROIs) in the neuroinflammatory process were a priori selected for comparison between psychotic patients and healthy volunteers. ${ }^{11} \mathrm{C}-(R)-\mathrm{PK} 11195$ time-activity curves of the frontal, occipital, temporal, and parietal lobes; basal ganglia; hippocampus; and cerebellum were created using an automated ROI tool. In addition, time-activity curves of the midbrain and brain stem were created using manually drawn ROIs. The timeactivity curves were used for kinetic modeling using software developed in MATLAB 7.1 (The MathWorks). The individual delay was calculated to correct for the delay in radioactivity measurements in blood, caused by the distance between the subject and the automated sampling system. Two-tissue-compartment modeling (Fig. 1) was used to calculate the $K_{1}-k_{4}$ using the metabolite-corrected plasma curve as an input function, correction for the individual delay, and an individually fixed blood volume, which was the median of the blood volume of all areas determined using 2-tissue-compartment modeling with a free blood volume. The fixed instead of the free blood volume was chosen because it provides a more stable measurement of $K_{1}-k_{4}$. The binding potential was defined as $k_{3} / k_{4}$ and was calculated for each ROI individually.

\section{Statistics}

Statistical analysis was performed with SPSS 16.0 (SPSS Inc.). One-way ANOVA was used to determine group differences between the tracer and subject characteristics and whole-brain gray matter binding potential. Statistical analysis on the binding potentials in the examined brain regions was performed using a multivariate general linear model, with the whole-brain gray matter binding potential as a covariate to correct for global ${ }^{11} \mathrm{C}$ (R)-PK11195 uptake. To correct for multiple comparisons, the $P$ value threshold was defined according to Bonferroni. Therefore, 0.05 was divided by the number of brain regions $(n=10)$, resulting in a significance threshold of 0.005 . Correlations between the score on the positive scale of the PANSS and binding potentials were assessed with the Pearson product moment correlation coefficient $(r)$ and were assumed to be significant when $P$ was less than 0.05 .

\section{RESULTS}

\section{Subjects}

Subject characteristics are displayed in Table 1. The diagnostic SCAN interview confirmed that all patients fit within the DSM-IV criteria of schizophrenia-spectrum

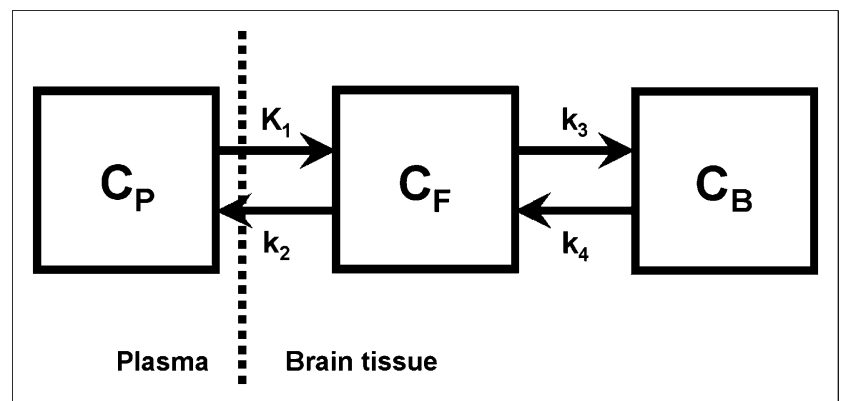

FIGURE 1. Two-tissue-compartment model to describe kinetics of ${ }^{11} \mathrm{C}-(R)-\mathrm{PK} 11195$. Influx of ${ }^{11} \mathrm{C}-(R)-\mathrm{PK} 11195$ from plasma $\left(\mathrm{C}_{\mathrm{P}}\right)$ to brain tissue is described by $K_{1}$, and efflux of ${ }^{11} \mathrm{C}-(R)$-PK11195 from brain tissue to plasma is described by $k_{2}$. In brain tissue, ${ }^{11} \mathrm{C}-(R)-\mathrm{PK} 11195$ can be either free $\left(\mathrm{C}_{\mathrm{F}}\right)$ or bound to peripheral benzodiazepine receptor $\left(\mathrm{C}_{\mathrm{B}}\right)$. Binding to receptor is described by $k_{3}$, and because ${ }^{11} \mathrm{C}-(R)$ PK11195 binds reversibly to PBR, it can return to free compartment, as described by $k_{4}$. Binding potential is defined as $k_{3} / k_{4}$. 
TABLE 1. Subject Characteristics

\begin{tabular}{|c|c|c|c|c|c|c|c|c|c|}
\hline Subject no. & Sex & Age $(\mathrm{y})$ & DSM-IV & $\begin{array}{l}\text { Onset first } \\
\text { psychosis }\end{array}$ & $\begin{array}{c}\text { No. of } \\
\text { psychoses }\end{array}$ & $\begin{array}{l}\text { Positive } \\
\text { PANSS }\end{array}$ & $\begin{array}{l}\text { Negative } \\
\text { PANSS }\end{array}$ & $\begin{array}{l}\text { Global } \\
\text { PANSS }\end{array}$ & Antipsychotic \\
\hline \multicolumn{10}{|c|}{ Healthy volunteers } \\
\hline 1 & $\mathrm{~F}$ & 27 & - & - & - & - & - & - & - \\
\hline 2 & $F$ & 39 & - & - & - & - & - & - & - \\
\hline 3 & $\mathrm{~F}$ & 23 & - & - & - & - & - & - & - \\
\hline 4 & M & 23 & - & - & - & - & - & - & - \\
\hline 5 & M & 24 & - & - & - & - & - & - & - \\
\hline 6 & M & 22 & - & - & - & - & - & - & - \\
\hline 7 & M & 30 & - & - & - & - & - & - & - \\
\hline 8 & M & 27 & - & - & - & - & - & - & - \\
\hline \multicolumn{10}{|l|}{ Patients } \\
\hline 1 & M & 28 & 295.30 & 21 & 4 & 20 & 21 & 40 & Olanzapine \\
\hline 2 & M & 42 & 298.90 & 26 & 2 & 20 & 10 & 28 & Zuclopentixol \\
\hline 3 & $\mathrm{~F}$ & 40 & 295.30 & 40 & 1 & 19 & 18 & 37 & Olanzapine \\
\hline 4 & $\mathrm{M}$ & 28 & 295.30 & 26 & 1 & 18 & 19 & 42 & Haloperidol \\
\hline 5 & M & 31 & 295.30 & 29 & 1 & 21 & 19 & 37 & Risperidone \\
\hline 6 & M & 28 & 295.30 & 20 & 2 & 25 & 20 & 47 & Risperidone \\
\hline 7 & $\mathrm{M}$ & 22 & 298.90 & 20 & 2 & 15 & 9 & 30 & Risperidone \\
\hline
\end{tabular}

disorders. Five patients were diagnosed as having schizophrenia of the paranoid type and 2 patients as having a brief psychotic disorder not otherwise specified. Six patients were scanned within 2 mo after onset of a psychotic episode, and 1 patient was chronically symptomatic. The number of lifetime psychotic episodes ranged from 1 to 4 . The average score was $20 \pm 3$ on the positive scale of the PANSS, $17 \pm 5$ on the negative scale, and $37 \pm 7$ on the global scale. No significant differences in age were found between patients $(31 \pm 7)$ and healthy volunteers $(27 \pm 6)$ $(P=0.124)$.

\section{${ }^{11} \mathrm{C}-(\mathrm{R})-\mathrm{PK} 11195$ PET}

A strong significantly higher ${ }^{11} \mathrm{C}-(R)$-PK11195 binding potential was found in the hippocampus of patients than in healthy volunteers $(2.07 \pm 0.42$ vs. $1.37 \pm 0.30 ; P=$ 0.004) (Table 2). The whole-brain gray matter (Fig. 2) and white matter binding potential of ${ }^{11} \mathrm{C}-(R)-\mathrm{PK} 11195$ were, respectively, $30 \%$ higher $(1.99 \pm 0.64$ vs. $1.54 \pm 0.41 ; P=$

\begin{tabular}{|c|c|c|c|}
\hline Region & Healthy volunteers & Patients & $P$ \\
\hline Frontal lobe & $1.76 \pm 0.75$ & $2.08 \pm 0.76$ & 0.459 \\
\hline Occipital lobe & $1.83 \pm 1.20$ & $1.93 \pm 0.74$ & 0.892 \\
\hline Temporal lobe & $1.28 \pm 0.34$ & $1.64 \pm 0.56$ & 0.079 \\
\hline Parietal lobe & $1.77 \pm 0.93$ & $2.28 \pm 1.22$ & 0.720 \\
\hline Basal ganglia & $1.39 \pm 0.28$ & $1.82 \pm 0.59$ & 0.017 \\
\hline Thalamus & $1.61 \pm 1.44$ & $1.49 \pm 0.35$ & 0.742 \\
\hline Hippocampus & $1.37 \pm 0.30$ & $2.07 \pm 0.42$ & $0.004^{*}$ \\
\hline Midbrain & $1.68 \pm 0.60$ & $2.63 \pm 0.40$ & 0.014 \\
\hline Cerebellum & $1.11 \pm 0.22$ & $1.45 \pm 0.48$ & 0.040 \\
\hline Pons & $1.54 \pm 0.32$ & $2.85 \pm 1.42$ & 0.027 \\
\hline \multicolumn{4}{|c|}{$\begin{array}{l}{ }^{\star} P<0.005 \text {. } \\
\text { Statistical analysis was performed using multivariate general } \\
\text { linear model, with whole-brain gray matter as covariate. Data are } \\
\text { average } \pm S D \text {. }\end{array}$} \\
\hline
\end{tabular}

$0.122)$ and $20 \%$ higher $(2.18 \pm 0.70$ vs. $1.73 \pm 0.51 ; P=$ 0.180 ) in patients than in healthy volunteers, but this difference did not reach statistical significance. A nonsignificant focal increase of ${ }^{11} \mathrm{C}-(R)$-PK11195 binding potential was found in the midbrain $(2.63 \pm 0.40$ vs. $1.68 \pm 0.60$; $P=0.014)$, basal ganglia $(1.82 \pm 0.59$ vs. $1.39 \pm 0.28 ; P=$ $0.017)$, pons ( $2.85 \pm 1.42$ vs. $1.54 \pm 0.32 ; P=0.027)$, and cerebellum $(1.45 \pm 0.48$ vs. $1.11 \pm 0.22 ; P=0.040)$ of patients when compared with healthy volunteers. No statistically significant differences were found in the influx of ${ }^{11} \mathrm{C}$ - $(R)$-PK11195 $\left(K_{1}\right)$ in any of the examined brain areas between patients and healthy volunteers. A significantly higher efflux $\left(k_{2}\right)$ of ${ }^{11} \mathrm{C}-(R)$-PK11195 was found in all examined brain areas in patients than in healthy volunteers $(P<0.005)$.

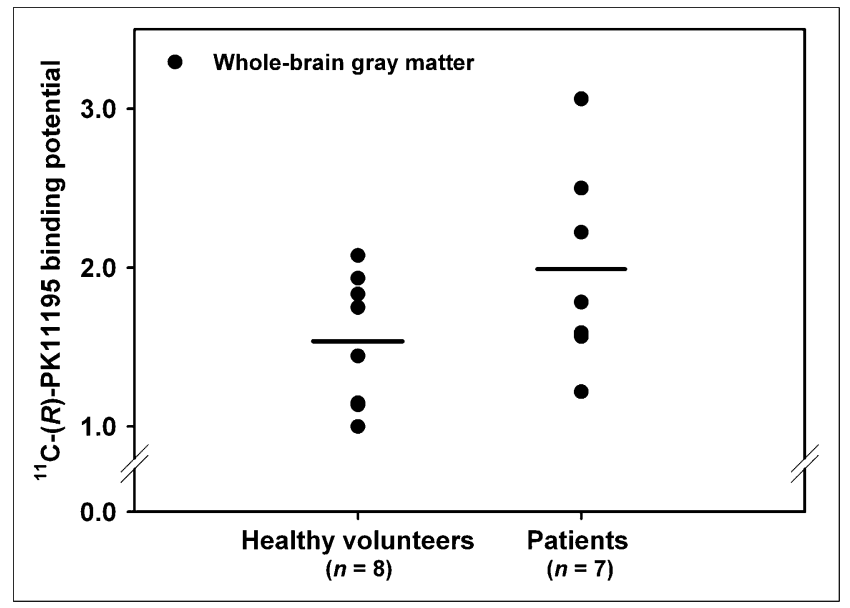

FIGURE 2. Binding potential of ${ }^{11} \mathrm{C}-(R)$-PK11195 in wholebrain gray matter. Each dot represents an individual subject, and horizontal lines represent mean values. 
No correlations were found between the total score on the positive, negative, and global scales of the PANSS with both the whole-brain gray matter and hippocampal binding potential. Age, the total number of psychotic episodes, the onset of the first psychotic episode, and the duration of the psychotic episodes were not correlated to the ${ }^{11} \mathrm{C}-(R)-$ PK11195 binding potential.

\section{MRI}

Visual examination of the MRI scans by an experienced neuroradiologist did not show any gross structural abnormalities in either patients or controls. In particular, no white matter lesions, indicative of neuroinflammation, were found.

\section{DISCUSSION}

In the present study, we have shown a statistically significant increased binding potential of ${ }^{11} \mathrm{C}-(R)-\mathrm{PK} 11195$ in the hippocampus of psychotic patients as compared with healthy volunteers, indicating the presence of neuroinflammation. To our knowledge, this is the first study that reveals the presence of neuroinflammation in a focal area in living patients during psychosis. The presence of focal neuroinflammation in the hippocampus was accompanied by a nonsignificant increase in the whole-brain gray and white matter ${ }^{11} \mathrm{C}-(R)-\mathrm{PK} 11195$ binding potential.

In a recent study, Van Berckel et al. (8) showed a small increase in the whole-brain gray matter binding potential of ${ }^{11} \mathrm{C}-(R)-\mathrm{PK} 11195$ in stable schizophrenic patients, with no reported focal increase. This finding is in line with the hypothesis of our study that focal neuroinflammation is a feature of psychosis and not necessarily present in stable schizophrenic patients. The focal neuroinflammation may evolve into a more widespread process in time. Increased focal neuroinflammation during psychosis is an important pathologic finding. The neuroinflammation found in the hippocampus may reflect the exceptional vulnerability of this region during psychosis.

The relation between schizophrenia and neuroinflammation was shown in several postmortem studies, demonstrating an increase in the number of activated microglia cells $(4,5,7)$, but other studies failed to show differences in the presence of activated microglia cells between the brain of schizophrenic patients and control subjects $(6,10-12)$. Steiner et al. (6) could not find support for the presence of activated microglia cells in schizophrenia, but 2 patients who died from suicide during acute psychosis showed highly elevated microglia cell activation. In a subsequent study, Steiner et al. (13) found a significantly increased density of activated microglia cells in the dorsolateral prefrontal cortex, nucleus accumbens, and mediodorsal thalamus of 6 schizophrenic patients who committed suicide during acute psychosis, compared with 10 nonsuicidal schizophrenic patients. A nonsignificant increased density of activated microglia cells was found in the hippocampus. No differences in the density of activated microglia cells were found between nonsuicidal schizo- phrenic patients and healthy volunteers. Although suicidal behavior may be linked to other regions of focal neuroinflammation, these results are consistent with the findings in the present study that focal neuroinflammation occurs predominately during psychosis.

Neuroinflammation is characterized by the activation of microglia cells; however, activated astrocytes also play an important role in the neuroinflammatory process (14). As in activated microglia cells, the expression of the PBR is also increased in activated astrocytes $(15-17)$. Increased ${ }^{11} \mathrm{C}$ $(R)$-PK11195 can therefore represent activated microglia cells or astrocytes. To date, the cell type that is responsible for the increased uptake of ${ }^{11} \mathrm{C}-(R)-\mathrm{PK} 11195$, and thus the increased expression of the PBR in neurologic disorders, is not exactly known. However, many studies are performed to unravel the contribution of activated microglia cells or astrocytes to the PBR-mediated ${ }^{11} \mathrm{C}-(R)$-PK11195 uptake. It has been shown that the contribution is dependent on the phase of activation of microglia cells and astrocytes (15) and on the type of lesion that is investigated. For example, in facial nerve axotomy, only microglia cells were responsible for the ${ }^{11} \mathrm{C}-(R)-\mathrm{PK} 11195$ signal (18), whereas in a toxic lesion of the hippocampus an increase in PBR expression was found in both microglia cells and astrocytes (19). However, it cannot be excluded that the increased ${ }^{11} \mathrm{C}$ (R)-PK11195 binding potential found in this study is due to activated astrocytes only, unrelated to a neuroinflammatory process. The calcium binding protein S100B, which is primarily expressed by activated astrocytes, was found to be increased in serum and plasma of schizophrenic patients without damage to neurons and astrocytes (20). However, because it is not known if S100B-expressing astrocytes also show an increase in PBR expression, and because it has been suggested that $\mathrm{S} 100 \mathrm{~B}$ contributes to neuroinflammation by activation of microglia cells (21), additional research is necessary to unravel the exact mechanism behind S100B expression in schizophrenia. The dopamine hypothesis of schizophrenia might give another alternative explanation for the increased expression of the PBR that was found in the present study. It has been reported that norepinephrine, dopamine, and serotonin increased the binding of the PBR ligand ${ }^{3} \mathrm{H}-\mathrm{Ro5}-4864$ to the PBR in cultured astrocytes (22). In the same study, however, it was reported that the binding of ${ }^{3} \mathrm{H}-\mathrm{PK} 11195$ was not affected by these neurotransmitters, which was ascribed to the presence of different binding sites for Ro5-4864 and PK11195. Taken together, regardless of activated microglia cells or astrocytes being responsible for the increased expression in the $\mathrm{PBR}$, the focal increased ${ }^{11} \mathrm{C}-(R)-\mathrm{PK} 11195$ in schizophrenia most likely represents a neuroinflammatory process.

In the present study, neuroinflammation was found in the hippocampus. Hippocampal pathology in schizophrenia is abundantly reported in the literature. MRI studies showed a decrease in hippocampal size in schizophrenic patients, even in prodromal and first-episode patients, demonstrating that it is not secondary to treatment $(23,24)$. Despite the 
reported decrease in hippocampal volume, there is no evidence for a change in the total number of neurons in the hippocampus of schizophrenia $(25,26)$. More likely, the morphology of the hippocampal neurons in schizophrenia is altered with respect to parameters such as size, shape, and organization (27). The involvement of microglia cells in hippocampal pathology was hitherto unknown.

The marked increase in ${ }^{11} \mathrm{C}-(R)-\mathrm{PK} 11195$ binding potential in the hippocampus suggests that neuroinflammation plays an important role in schizophrenia, especially during psychosis, and provides a potential target for therapy. In fact, it was shown that both patients with an acute exacerbation of schizophrenia (28) and patients with chronic schizophrenia (29) who were treated with the cyclooxygenase- 2 inhibitor celecoxib in addition to risperidone had a significantly greater improvement on the PANSS than did the patients who were treated with risperidone alone. In addition, the broad-spectrum tetracycline antibiotic minocycline was found to be beneficial as an add-on treatment in schizophrenia, in both an open-label study (30) and a double-blind placebo-controlled study (31). Besides celecoxib and minocycline, the addition of aspirin to regular antipsychotic treatment over a period of 3 mo was also found to substantially reduce the symptoms of schizophrenia, compared with the treatment of patients with antipsychotics only (32). Thus, the antiinflammatory drugs celecoxib, minocycline, and aspirin can improve the symptoms of schizophrenia, most likely because of the inhibition of proinflammatory cytokines. These results are in agreement with the presence of a neuroinflammatory process in schizophrenia.

Because all patients were taking antipsychotic medication during the period in which the ${ }^{11} \mathrm{C}-(R)-\mathrm{PK} 11195$ PET scan was obtained, there is a possibility that the medication influenced the microglia cell activation. In vitro studies on the effect of antipsychotic drugs on microglia cells show that they decrease the neurotoxic molecules released by activated microglia cells. Both risperidone and haloperidol were found to inhibit the production of nitric oxide, the expression of inducible nitric oxide synthase, and the production of proinflammatory cytokines by microglia cells treated with interferon- $\gamma(33)$. Olanzapine was also found to have antiinflammatory properties, because the production of nitric oxide by lipopolysaccharide-stimulated microglia cells was reduced by pretreatment with olanzapine (34). In addition to the in vitro studies, we have recently shown that both clozapine and risperidone inhibited the activation of microglia cells in a rat model of herpes simplex encephalitis, whereas chronic treatment with clozapine and risperidone of healthy rats did not cause activation of microglia cells (data will be published elsewhere). Thus, antipsychotic drugs most likely affect neuroinflammation in such a way that they decrease the microglia cell activation rather than cause activation of the microglia cells.

In addition to antipsychotics, all patients have used benzodiazepines. Because benzodiazepines were found to have affinity for the PBR, the patients had to stop treatment with benzodiazepines at a minimum of 3 half-lives (on average, 1-2 wk) of the benzodiazepines before the start of the study, to prevent a direct effect on the binding of ${ }^{11} \mathrm{C}$ (R)-PK11195. It cannot, however, be ruled out that treatment with benzodiazepines affected the expression of the PBR. Most studies on benzodiazepines and other PBR ligands, such as Ro5-4864 and PK11195, indicate the neuroprotective effects of these ligands (35). Wilms et al. (36) have shown that midazolam, clonazepam, and diazepam interfered with the in vitro synthesis and release of proinflammatory cytokines by microglia cells. In vivo, it has been shown that diazepam protected against neuronal death in the hippocampus after transient forebrain ischemia (37). Benzodiazepines, like antipsychotics, decreased neuroinflammation rather than were responsible for the increased ${ }^{11} \mathrm{C}-(R)-\mathrm{PK} 11195$ binding potential in the hippocampus. However, additional research on the role of both antipsychotics and benzodiazepines is necessary to determine their (protective) role in neuroinflammation.

\section{CONCLUSION}

The present study in patients within the schizophrenia spectrum suggests the presence of focal neuroinflammation in the hippocampus during psychosis. This focal neuroinflammation was not accompanied by structural abnormalities. Which cell type is responsible for the increased ${ }^{11} \mathrm{C}-(R)-\mathrm{PK} 11195$ binding potential and how neuroinflammation is related to psychosis in schizophrenia remains to be clarified. Therefore, future studies are needed for the further evaluation of the role of focal neuroinflammation during psychosis and the feasibility of antiinflammatory drugs to treat the disease.

\section{ACKNOWLEDGMENTS}

We thank Rixt Elgersma and the nurse staff of Lentis Winschoten, The Netherlands, for accompanying the patients. This study was funded by the Stanley Medical Research Institute, grant-ID 05-NV-001.

\section{REFERENCES}

1. Muller N, Schwarz MJ. A psychoneuroimmunological perspective to Emil Kraepelins dichotomy: schizophrenia and major depression as inflammatory CNS disorders. Eur Arch Psychiatry Clin Neurosci. 2008;258:97-106.

2. Nimmerjahn A, Kirchhoff F, Helmchen F. Resting microglial cells are highly dynamic surveillants of brain parenchyma in vivo. Science. 2005;308:13141318

3. Doorduin J, de Vries EFJ, Dierckx RA, Klein HC. PET imaging of the peripheral benzodiazepine receptor: monitoring disease progression and therapy response in neurodegenerative disorders. Curr Pharm Des. 2008;14:3297-3315.

4. Bayer TA, Buslei R, Havas L, Falkai P. Evidence for activation of microglia in patients with psychiatric illnesses. Neurosci Lett. 1999;271:126-128.

5. Radewicz K, Garey LJ, Gentleman SM, Reynolds R. Increase in HLA-DR immunoreactive microglia in frontal and temporal cortex of chronic schizophrenics. J Neuropathol Exp Neurol. 2000;59:137-150.

6. Steiner J, Mawrin C, Ziegeler A, et al. Distribution of HLA-DR-positive microglia in schizophrenia reflects impaired cerebral lateralization. Acta Neuropathol. 2006;112: 305-316. 
7. Wierzba-Bobrowicz T, Lewandowska E, Lechowicz W, Stepien T, Pasennik E. Quantitative analysis of activated microglia, ramified and damage of processes in the frontal and temporal lobes of chronic schizophrenics. Folia Neuropathol. 2005;43:81-89.

8. van Berckel BN, Bossong MG, Boellaard R, et al. Microglia activation in recentonset schizophrenia: a quantitative $(R)-\left[{ }^{11} \mathrm{C}\right] \mathrm{PK} 11195$ positron emission tomography study. Biol Psychiatry. 2008;64:820-822.

9. American Psychiatric Association. Diagnostic and Statistical Manual of Mental Disorders: DSM-IV. 4th ed. Washington, DC: American Psychiatric Association; 1994.

10. Arnold SE, Trojanowski JQ, Gur RE, Blackwell P, Han LY, Choi C. Absence of neurodegeneration and neural injury in the cerebral cortex in a sample of elderly patients with schizophrenia. Arch Gen Psychiatry. 1998;55:225-232.

11. Falke E, Han LY, Arnold SE. Absence of neurodegeneration in the thalamus and caudate of elderly patients with schizophrenia. Psychiatry Res. 2000;93:103110 .

12. Kurumaji A, Wakai T, Toru M. Decreases in peripheral-type benzodiazepine receptors in postmortem brains of chronic schizophrenics. J Neural Transm. 1997; 104:1361-1370.

13. Steiner J, Bielau H, Brisch R, et al. Immunological aspects in the neurobiology of suicide: elevated microglial density in schizophrenia and depression is associated with suicide. J Psychiatr Res. 2008;42:151-157.

14. Chavarria A, Alcocer-Varela J. Is damage in central nervous system due to inflammation? Autoimmun Rev. 2004;3:251-260.

15. Chen MK, Guilarte TR. Translocator protein $18 \mathrm{kDa}$ (TSPO): molecular sensor of brain injury and repair. Pharmacol Ther. 2008;118:1-17.

16. Itzhak Y, Baker L, Norenberg MD. Characterization of the peripheral-type benzodiazepine receptors in cultured astrocytes: evidence for multiplicity. Glia. 1993;9:211-218.

17. Raghavendra Rao VL, Dogan A, Bowen KK, Dempsey RJ. Traumatic brain injury leads to increased expression of peripheral-type benzodiazepine receptors, neuronal death, and activation of astrocytes and microglia in rat thalamus. Exp Neurol. 2000;161:102-114

18. Banati RB, Myers R, Kreutzberg GW. PK ('peripheral benzodiazepine')-binding sites in the CNS indicate early and discrete brain lesions: microautoradiographic detection of $\left[{ }^{3} \mathrm{H}\right] \mathrm{PK} 11195$ binding to activated microglia. J Neurocytol. 1997;26: $77-82$.

19. Kuhlmann AC, Guilarte TR. Cellular and subcellular localization of peripheral benzodiazepine receptors after trimethyltin neurotoxicity. J Neurochem. 2000; 74:1694-1704.

20. Steiner J, Bielau H, Bernstein HG, Bogerts B, Wunderlich MT. Increased cerebrospinal fluid and serum levels of S100B in first-onset schizophrenia are not related to a degenerative release of glial fibrillar acidic protein, myelin basic protein and neurone-specific enolase from glia or neurones. J Neurol Neurosurg Psychiatry. 2006;77:1284-1287.

21. Rothermundt M, Peters M, Prehn JH, Arolt V. S100B in brain damage and neurodegeneration. Microsc Res Tech. 2003;60:614-632.
22. Itzhak Y, Norenberg MD. Regulation of peripheral-type benzodiazepine receptors in cultured astrocytes by monoamine and amino acid neurotransmitters. Brain Res. 1994;660:346-348.

23. Pantelis C, Velakoulis D, McGorry PD, et al. Neuroanatomical abnormalities before and after onset of psychosis: a cross-sectional and longitudinal MRI comparison. Lancet. 2003;361:281-288.

24. Wright IC, Rabe-Hesketh S, Woodruff PW, David AS, Murray RM, Bullmore ET. Meta-analysis of regional brain volumes in schizophrenia. Am J Psychiatry. 2000;157:16-25.

25. Heckers S, Heinsen H, Geiger B, Beckmann H. Hippocampal neuron number in schizophrenia: a stereological study. Arch Gen Psychiatry. 1991;48:1002-1008.

26. Walker MA, Highley JR, Esiri MM, et al. Estimated neuronal populations and volumes of the hippocampus and its subfields in schizophrenia. Am J Psychiatry. 2002; 159:821-828.

27. Harrison PJ. The hippocampus in schizophrenia: a review of the neuropathological evidence and its pathophysiological implications. Psychopharmacology (Berl). 2004;174:151-162.

28. Muller N, Riedel M, Scheppach C, et al. Beneficial antipsychotic effects of celecoxib add-on therapy compared to risperidone alone in schizophrenia. Am J Psychiatry. 2002;159:1029-1034.

29. Akhondzadeh S, Tabatabaee M, Amini H, Ahmadi Abhari SA, Abbasi SH, Behnam B. Celecoxib as adjunctive therapy in schizophrenia: a double-blind, randomized and placebo-controlled trial. Schizophr Res. 2007;90:179-185.

30. Miyaoka T, Yasukawa R, Yasuda H, Hayashida M, Inagaki T, Horiguchi J. Minocycline as adjunctive therapy for schizophrenia: an open-label study. Clin Neuropharmacol. 2008;31:287-292.

31. Levkovitz Y, Mendlovic S, Issaki S, Braw Y, Levkovitch-Verbin H, Gal G. Minocycline ameliorates negative symptoms and cognitive deficits in earlyphase schizophrenia: a double-blind, randomized placebo-controlled study. Schizophr Res. 2008;98(suppl 1):14.

32. Laan W. Anti-Inflammatory Drugs and Psychosis [thesis]. University of Utrecht, Utrecht, The Netherlands; 2008.

33. Kato T, Monji A, Hashioka S, Kanba S. Risperidone significantly inhibits interferon- $\gamma$-induced microglial activation in vitro. Schizophr Res. 2007;92: $108-115$.

34. Hou Y, Wu CF, Yang JY, et al. Effects of clozapine, olanzapine and haloperidol on nitric oxide production by lipopolysaccharide-activated N9 cells. Prog Neuropsychopharmacol Biol Psychiatry. 2006;30:1523-1528.

35. Papadopoulos V, Lecanu L. Translocator protein (18 kDa) TSPO: an emerging therapeutic target in neurotrauma. Exp Neurol. 2009;219:53-57.

36. Wilms H, Claasen J, Rohl C, Sievers J, Deuschl G, Lucius R. Involvement of benzodiazepine receptors in neuroinflammatory and neurodegenerative diseases: evidence from activated microglial cells in vitro. Neurobiol Dis. 2003;14:417-424.

37. Sarnowska A, Beresewicz M, Zablocka B, Domanska-Janik K. Diazepam neuroprotection in excitotoxic and oxidative stress involves a mitochondrial mechanism additional to the GABAAR and hypothermic effects. Neurochem Int. 2009;55:164-173. 